ORIGINAL ARTICLE

\title{
Outpatient diagnosis of pulmonary embolism: the MIOPED (Manchester Investigation Of Pulmonary Embolism Diagnosis) study
}

\author{
K Hogg, D Dawson, K Mackway-Jones
}

Emerg Med J 2006;23:123-127. doi: 10.1136/emj.2005.027110

Background and objectives: Pleuritic chest pain, a symptom of pulmonary embolism, is a common presenting symptom in the emergency department. The aim of this study was to validate an algorithm for the diagnosis of pulmonary embolism in emergency department patients with pleuritic chest pain.

Methods: This was a prospective, diagnostic cohort study conducted in a large UK city centre emergency department. A total of 425 patients with pleuritic chest pain presenting to the emergency department between February 2002 and June 2003 were recruited. Patients scoring a low modified Wells clinical

See end of article for authors' affiliations probability of pulmonary embolism, who had a normal latex agglutination D-dimer, were discharged. All others followed a diagnostic imaging protocol to exclude and diagnose pulmonary embolism using PIOPED interpreted ventilation-perfusion scanning, CT pulmonary angiography, and digital subtraction pulmonary angiography. All patients were followed up for three months for evidence of pulmonary embolism or deep vein thrombosis. An independent adjudication committee reviewed all deaths.

Results: A total of 408 patients completed the diagnostic algorithm; 86.5\% (353/408) were investigated as outpatients, $5.4 \%(22 / 408)$ were diagnosed as having pulmonary embolism, and $98.8 \%(403 / 408)$ were followed up for three months. Of the 381 patients without pulmonary embolism who completed follow up, the incidence of thromboembolic disease was $0.8 \%(95 \% \mathrm{Cl} 0.3 \%$ to $2.3 \%$ ): two patients had pulmonary embolism and one had a deep vein thrombosis.

Conclusions: The MIOPED (Manchester Investigation Of Pulmonary Embolism Diagnosis) diagnostic protocol can safely exclude pulmonary embolism in outpatients with pleuritic chest pain.
$M$ ortality from pulmonary embolic disease has remained unchanged during the past 25 years. ${ }^{12}$ By the age of $80,10.7 \%$ of the population will have experienced a thromboembolic event. ${ }^{3}$ Improved diagnosis is the first step to reducing morbidity and mortality.

Pulmonary embolism can cause pleuritic chest pain. ${ }^{45}$ Pleuritic chest pain accounts for $1 \%$ of attendances to emergency departments ( $\mathrm{J}$ Wright et al, unpublished observation, 2003) and around 170000 emergency department patients per year in England alone. Simple chest radiography will exclude the diagnosis of pneumothorax, but there is no single test which can exclude a pulmonary embolus. A patient with a pulmonary embolus can have a normal blood gas analysis, ${ }^{6} \mathrm{ECG},{ }^{7}$ and chest $x$ ray. ${ }^{8}$

The diagnosis of pulmonary embolism has undergone several fundamental changes in the past decade. The combination of D-dimer and clinical probability now replaces ventilation-perfusion scanning as first line testing. ${ }^{9-11}$ The Prospective Investigation Of Pulmonary Embolism Diagnosis (PIOPED) study ${ }^{12}$ showed that combining a standardised assessment of the ventilation-perfusion scan with clinical probability will accurately exclude and diagnose pulmonary embolism in a proportion of patients. Computed tomography (CT) pulmonary angiography is highly specific for pulmonary embolism. ${ }^{13-15}$ Despite these advances there are few guidelines on how best to combine the individual tests to exclude and diagnose pulmonary embolism in every patient.

This study aimed to establish the safety of a diagnostic algorithm combining clinical probability, D-dimer, ventilation-perfusion scanning, CT pulmonary angiography and digital subtraction pulmonary angiography in patients with pleuritic chest pain.

\section{METHODS \\ Patients}

The Central Manchester Local Research Ethics Committee (reference CEN/00/082) approved the study. The Manchester Investigation Of Pulmonary Embolism Diagnosis (MIOPED) study was a single centre, prospective diagnostic study with the primary aim of assessing the utility of bedside tests for pulmonary embolism. The data reported in this paper are extracted from the MIOPED database. The methods have been previously reported. ${ }^{16}$ In brief, patients with objectively defined pleuritic chest pain presenting to Manchester Royal Infirmary's emergency department were prospectively consented to the study. Exclusion criteria were trauma, pregnancy, pneumothorax, myocardial infarction, cardiac ischaemia or pericarditis, hypoxia with $\mathrm{PaO}_{2}<7.5 \mathrm{kPa}$, age $<18$ years, contraindication to contrast investigations and weight $>140 \mathrm{~kg}$. All emergency department notes were reviewed. Details were taken from the notes of any patient who was not recruited and who described chest pain as sharp or pleuritic.

\section{Clinical probability and D-dimer}

All patients were assigned a modified Wells clinical probability of pulmonary embolism by an emergency department physician. The modified Wells score adds intravenous drug use as 3.0 points (S Jones et al, unpublished observation, 2001) to Wells score. ${ }^{17}$ All patients underwent an IL Test D-dimer (Instrumentation Laboratory, Aragón, Barcelona, Spain). The MDA D-dimer (Organon Teknika BV, Boseind 15, Boxtel, the Netherlands) was used for four study patients. Figure 1 shows the diagnostic algorithm for pulmonary embolism. Patients with a low clinical probability and normal D-dimer had pulmonary embolism excluded. All others required diagnostic imaging. 


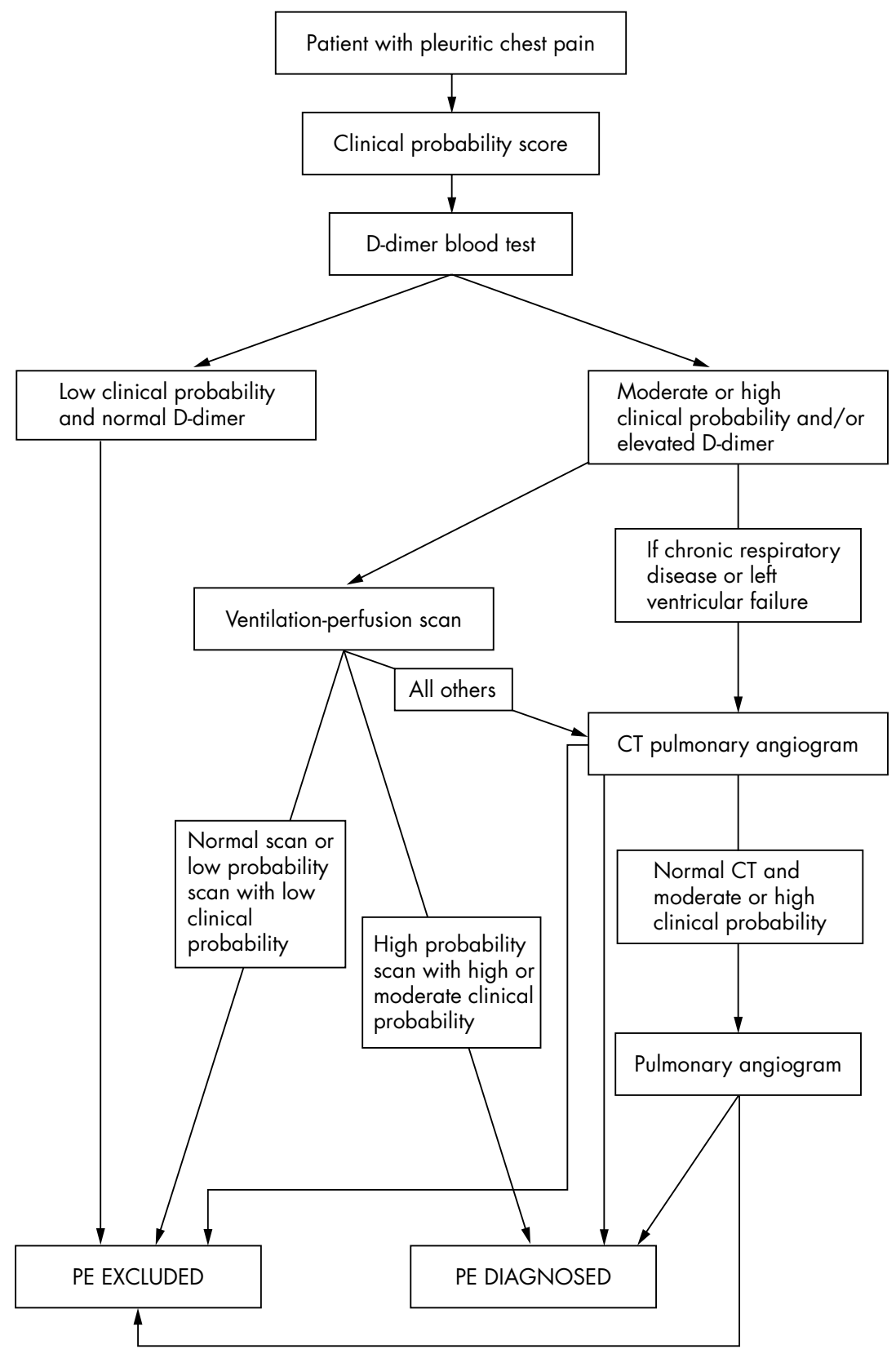

Figure 1 The diagnostic protocol. CT, computed tomography; PE, pulmonary embolism.

\section{Diagnostic imaging}

In patients who were ambulatory with oxygen saturations $>95 \%$ in air, respiratory rate $<20$ breaths per minute and heart rate $<100$ beats per minute after analgesia, diagnostic imaging was conducted as an outpatient. All patients were administered a daily dose of low molecular weight heparin during diagnostic imaging.

Ventilation-perfusion scans were interpreted using PIOPED criteria. ${ }^{12}$ A combination of low clinical probability and low probability ventilation-perfusion scan result excluded pulmonary embolic disease. A combination of moderate or high clinical probability and a high probability ventilation-perfusion scan result was diagnostic for pulmonary embolism. A normal or near normal scan excluded pulmonary embolism regardless of the clinical probability. All other result combinations were non-diagnostic and the patient had a CT pulmonary angiogram.

For patients with chronic respiratory disease or pulmonary oedema, CT pulmonary angiography was the primary diagnostic imaging technique. Patients with a normal CT pulmonary angiogram and low clinical probability had pulmonary embolism excluded. Patients with another pathology on CT had pulmonary embolism excluded. Those with a normal CT and moderate or high clinical probability underwent digital subtraction pulmonary angiography. All diagnostic images were retrospectively double checked for the MIOPED study, however for the purposes of this analysis only the initial (real-time) reports have been used.

The MIOPED study did not use lower limb ultrasound as a diagnostic test. The investigators sought to establish which patients with pleuritic chest pain had pulmonary embolism rather than deep vein thrombosis.

\section{Follow up}

All patients recruited into the study received written instructions to make contact if they experienced any further symptoms of chest pain, shortness of breath, or pain or swelling of the legs. The patients could page the lead 
researcher 24 hours a day. In addition, all patients were contacted by telephone three months after recruitment. Patients were followed up for evidence of deep vein thrombosis or pulmonary embolism.

\section{Adjudication committee}

An independent adjudication committee (two respiratory consultant physicians, one consultant in nuclear medicine, and one consultant radiologist) reviewed all cases in which a patient died during the study follow up. The committee also reviewed any case where a patient had undergone further testing for pulmonary embolism during the clinical follow up period, without the knowledge of the study group. The committee reviewed all hospital notes, ventilation-perfusion scans, CT scans, and postmortem reports. A consensus opinion was given on whether the patient's death or continued symptoms were "unlikely", "possibly", or "probably" caused by thromboembolic disease.

\section{Statistics}

A sample size of 400 patients was calculated. Assuming a pretest probability of $5 \%$ (95\% CI $3.3 \%$ to $7.6 \%)$, this sample size could demonstrate a reduction in probability to $1.1 \%$ (95\% CI $0.4 \%$ to $2.7 \%)$ post test. The Wilson "score" method ${ }^{18}{ }^{19}$ was used to calculate the $95 \%$ confidence interval around a proportion. All prospectively accumulated information was stored anonymously in a database using SPSS. Confidence intervals were calculated using StatsDirect (StatsDirect Ltd, Gresham Way, Sale, Cheshire, UK).

\section{RESULTS}

Between 11 February 2002 and 29 May 2003, 799 patients were assessed for inclusion into the MIOPED study. Of these, 97 declined to participate, 277 were excluded ( 171 because their pain did not fit our criteria for pleuritic chest pain), and 425 consented to participate in the study. During the same time period, an additional 633 patients attended the emergency department with chest pain described in the notes as either pleuritic or sharp. Table 1 compares the demographics of the patients who were recruited with those who declined, were excluded, or were not approached to be part of the study. Tables 2 and 3 show the MIOPED cohort risk factors for pulmonary embolism and examination findings.

A total of 408 patients completed the diagnostic algorithm correctly. Of these, 22 were diagnosed with pulmonary embolism (5.4\%), and 386 patients had pulmonary embolism excluded $(\mathrm{n}=229$ : normal $\mathrm{D}$-dimer and low clinical probability; $\mathrm{n}=157$ : patients following diagnostic imaging).

Table 1 Comparison of baseline characteristics

\begin{tabular}{|c|c|c|}
\hline & $\begin{array}{l}\text { Recruited } \\
\text { patients } \\
(n=425)\end{array}$ & $\begin{array}{l}\text { Patients not } \\
\text { recruited } \\
\text { ( } \mathrm{n}=1007 \text { ) }\end{array}$ \\
\hline Age in years (mean (SD)) & $38.3(15.0)$ & $40.2(17.9)$ \\
\hline \multicolumn{3}{|l|}{ Sex (\%) } \\
\hline Men & 48.9 & 49.0 \\
\hline Women & 51.1 & 51.0 \\
\hline \multicolumn{3}{|l|}{$\begin{array}{l}\text { Clinical probability of pulmonary } \\
\text { embolism (\%) }\end{array}$} \\
\hline Low & 86.6 & $84.1^{*}$ \\
\hline Medium & 10.1 & $13.0^{*}$ \\
\hline High & 3.3 & $2.8^{*}$ \\
\hline White cell count, $\times 10^{9} / /$ (mean (SD)) & $8.9(3.9)$ & $9.0 \times 10^{9} / \mathrm{L}(5.1) \ddagger$ \\
\hline $\mathrm{PaO}_{2}, \mathrm{kPa}($ mean $(\mathrm{SD}))$ & $11.8(2.2)$ & $11.0(4.5) \S$ \\
\hline $\mathrm{PaCO}_{2}, \mathrm{kPa}($ mean $(\mathrm{SD}))$ & $5.1(0.7)$ & $5.1(0.8)^{\oplus}$ \\
\hline
\end{tabular}

*Clinical probability was not possible to calculate in 862 patients. $\ddagger$ No white cell count taken on 203 patients.

§No arterial oxygen value measured for 857 patients.

-No arterial carbon dioxide values measured for 856 patients.

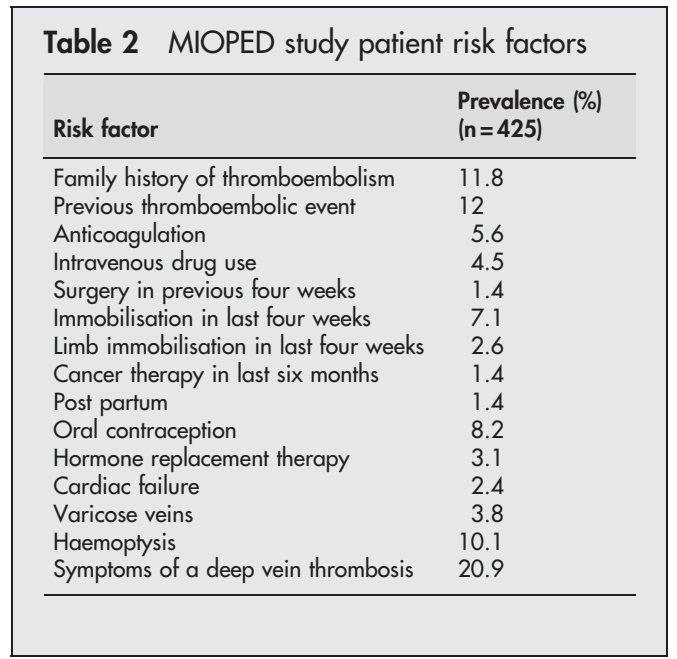

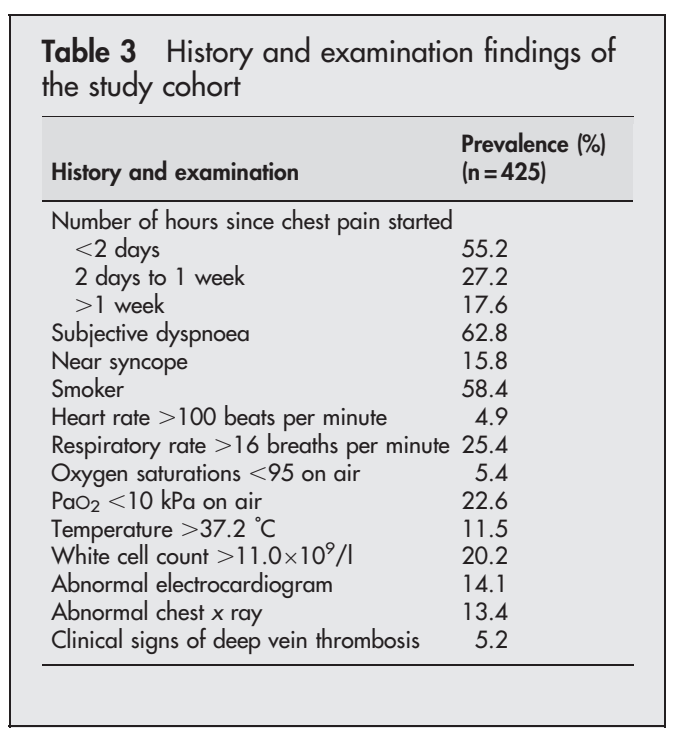

We performed 168 ventilation-perfusion scans (44 on the day of presentation, 74 on outpatients, 50 on inpatients), 79 CT scans ( 4 on the day of presentation, 32 on outpatients, 43 on inpatients), and 11 digital subtraction pulmonary angiograms ( 4 on outpatients, 7 on inpatients). Two hundred and seventy four patients $(67.2 \%)$ had pulmonary embolism diagnosed or excluded within one working day by the emergency department. An additional 79 patients (19.4\%) had diagnostic imaging as outpatients and 55 (13.5\%) as inpatients. All patients investigated as outpatients had an uncomplicated recovery.

Five $(1.2 \%)$ out of the 425 patients recruited were lost to follow up. Three hundred and sixty seven patients $(86.4 \%)$ were contacted directly by telephone and one by letter only. Twenty three next of kin (5.4\%) were asked about the study patient, and general practitioners provided information about 24 patients $(5.6 \%)$. A hospital consultant provided information about one patient. Thirty six patients were reviewed for a second time in the emergency department during follow up. Four patients died during follow up.

Three patients had a thromboembolic event during the three month follow up period. A 40 year old man returned to the emergency department with bilateral chest pain and haemoptysis, and a digital subtraction pulmonary angiogram 18 days after recruitment revealed bilateral pulmonary emboli. The adjudication committee deemed it "possible" the patient had pulmonary emboli on initial presentation. A 28 year old intravenous drug user was diagnosed with deep 
vein thrombosis two and a half months after recruitment. A 68 year old lady re-presented a week after recruitment with the same pleuritic chest pain. A ventilation-perfusion scan demonstrated a high probability of pulmonary embolic disease. The research team was not alerted to her return and the patient failed to undergo CT pulmonary angiography. The independent adjudication committee deemed it "possible" that she had pulmonary embolic disease.

All four deaths were reviewed by the adjudication committee. One was the patient described above, diagnosed with pulmonary embolism on digital subtraction pulmonary angiography 18 days after recruitment. The remaining deaths were deemed by the committee to be unrelated to pulmonary embolism.

Thus, of 381 patients who had pulmonary embolism excluded and completed follow up, the incidence of thromboembolic disease was $0.8 \%$ ( $95 \%$ CI $0.3 \%$ to $2.3 \%$ ).

\section{DISCUSSION}

The diagnostic algorithm presented here excluded pulmonary embolism in $94.6 \%$ of emergency department patients with pleuritic chest pain. Investigations in $87 \%$ of our cohort were conducted as outpatients. The three month incidence of thromboembolism in patients who had pulmonary embolism excluded was $0.8 \%$ ( $95 \%$ CI $0.3 \%$ to $2.3 \%$ ).

Pleuritic chest pain is a feature in $77 \%$ of patients with pulmonary embolism without underlying cardiorespiratory disease..$^{20}$ It is also a feature of pneumothorax, pericarditis, viral pleurisy, and atypical chest pain. Most of our patients were young with normal findings on chest $x$ rays and normal gas exchange. This makes it all the more difficult to establish which patients should undergo diagnostic imaging and which patients should not. Our study demonstrates that one in 20 of these outpatients will have an embolus (around 8500 per year in England). Many patients diagnosed as having embolic disease scored a low clinical probability of pulmonary embolism. A structured diagnostic approach enabled identification of those with embolism and avoided incorrect diagnosis and warfarinisation.

Despite all attempts, the study investigators were unable to recruit every patient who presented with pleuritic chest pain in the 16 month period. It was impossible to determine retrospectively if a patient's chest pain fitted our inclusion criteria and for that reason, all patients with chest pain documented as pleuritic or sharp were included as a potential missed patient. The MIOPED cohort was similar in age and sex distribution to those not recruited. A minority had clinical probability scoring or blood gas analysis, however it appears that those who did had similar characteristics to the MIOPED cohort. The study aimed to assess this diagnostic algorithm in a working environment. The doctors who interpreted the scans were the same doctors who normally report these investigations. No special efforts were made to influence the accuracy of the daily scan reporting, so the study results are a true representation of the working protocol.

The MIOPED study is the first study to propose this unique combination of D-dimer, clinical probability, ventilationperfusion scanning, CT pulmonary angiography, and digital subtraction angiography. One other study ${ }^{21}$ has assessed a diagnostic strategy in unselected outpatients with pleuritic chest pain. All 173 patients with pleuritic chest pain underwent ventilation-perfusion scanning. Those with an abnormal perfusion scan had pulmonary angiography. The three month false negative rate for pulmonary embolism was similar at $0.8 \%$ (95\% CI $0.1 \%$ to $4.6 \%$ ). The MIOPED study offers a less invasive diagnostic strategy which excluded pulmonary embolism in $53.9 \%$ of patients without diagnostic imaging.
During the MIOPED three month follow up, one patient was diagnosed as having pulmonary embolism on digital subtraction pulmonary angiography after a negative CT pulmonary angiogram. The adjudication committee deemed it "possible" the patient had pulmonary embolism on presentation. A second patient re-presented with a deep vein thrombosis two and a half months after recruitment. The original pleuritic chest pain was caused by a subpectoral abscess abutting the pleura (the swelling evident on the anterior chest wall, visualised on CT, and aspirated). It is doubtful that the deep vein thrombosis represented a missed pulmonary embolus at presentation. The last patient diagnosed as having pulmonary embolism during follow up failed to correctly complete the diagnostic algorithm. The adjudication committee deemed pulmonary embolism "possible" and our study results include this patient as a false negative result. In summary, our estimate of a $0.8 \%$ false negative rate may be an overestimation.

The MIOPED study has verified that an outpatient diagnostic protocol can safely exclude pulmonary embolism in patients presenting to the emergency department with pleuritic chest pain. Screening with a latex agglutination Ddimer and clinical probability allowed safe exclusion of the disease in the emergency department. Ventilation-perfusion scanning was invaluable as a low radiation imaging technique which was diagnostic in 110 out of 168 patients. Seventy nine CT pulmonary angiograms were performed diagnosing 17 cases of pulmonary embolism, 24 pneumonias (10 of whom had normal findings on chest $x$ rays), 2 carcinomas, and 3 cases of pulmonary abscess. Pulmonary angiography diagnosed pulmonary embolism in one patient who could not hold his breath for the CT scan.

Since this study was conducted, most hospitals have installed multislice CT scanners with improved diagnostic performance. Further research may show this reduces the diagnostic false negative rate.

\section{AUTHORS' CONTRIBUTORS}

$\mathrm{KH}$ designed and oversaw the study, recruited patients, analysed the data, and drafted the paper. DD recruited patients, maintained the database, and analysed the data. KMJ supervised the study. DD and KMJ redrafted the paper and approved the final version. There is noone else who fulfils the criteria of authorship. Acknowledgement: Magnus Harrison helped initiate the study.

$\mathrm{KH}$ is the guarantor for the paper.

\section{Authors' affiliations \\ K Hogg, D Dawson, K Mackway-Jones, Emergency Medicine Research Group, Manchester Royal Infirmary, Manchester, UK}

A part time study research nurse was funded by Pharmacia Limited for 13 months of the study. The study was conducted independent of the funding organisation.

Competing interests: Professor K Mackway-Jones is an editor for the Emergency Medicine Journal.

\section{REFERENCES}

1 Alpert J, Smith R, Carlson J, et al. Mortality in patients treated for pulmonary embolism. JAMA 1976;236:1477-80.

2 Goldhaber SZ, Visani L, De Rosa M. Acute pulmonary embolism: clinical outcomes in the International Cooperative Pulmonary Embolism Registry (ICOPER). Lancet 1999;353:1386-9.

3 Hansson PO, Welin L, Tibblin G, et al. Deep vein thrombosis and pulmonary embolism in the general population. "The Study of Men Born in 1913". Arch Intern Med 1997; 157:1665-70.

4 Miniati M, Prediletto R, Formichi B, et al. Accuracy of clinical assessment in the diagnosis of pulmonary embolism. Am J Respir Crit Care Med 1999;159:864-71

5 Stein PD, Willis PW III, DeMets DL. History and physical examination in acute pulmonary embolism in patients without pre-existing cardiac or pulmonary disease. Am J Cardiol 1981;47:218-23.

6 Stein PD, Goldhaber SZ, Henry JW, et al. Arterial blood gas analysis in the assessment of acute pulmonary embolism. Chest 1996;109:78-81. 
7 Rodger M, Makropoulos D, Turek M, et al. Diagnostic value of the electrocardiogram in suspected pulmonary embolism. Am J Cardiol 2000;86:807-9.

8 Elliott CG, Goldhaber SZ, Visani L, et al. Chest radiographs in acute pulmonary embolism. Results from the International Cooperative Pulmonary Embolism Registry. Chest 2000;118:33-8.

9 Kruip MJ, Slob MJ, Schijen $\mathrm{JH}$, et al. Use of a clinical decision rule in combination with $D$-dimer concentration in diagnostic workup of patients with suspected pulmonary embolism: a prospective management study. Arch Intern Med 2002;162:1631-5.

10 Wells PS, Anderson DR, Rodger M, et al. Excluding pulmonary embolism at the bedside without diagnostic imaging: management of patients with suspected pulmonary embolism presenting to the emergency department by using a simple clinical model and d-dimer. Ann Intern Med 2001;135:98-107.

11 Ginsberg JS, Wells PS, Kearon C, et al. Sensitivity and specificity of a rapid whole-blood assay for D-dimer in the diagnosis of pulmonary embolism. Ann Intern Med 1998; 129:1006-11.

12 The PIOPED Investigators. Value of the ventilation/perfusion scan in acute pulmonary embolism. Results of the Prospective Investigation of Pulmonary Embolism Diagnosis (PIOPED). JAMA 1990;263:2753-9.

13 Qanadli SD, Haijam ME, Mesurolle B, et al. Pulmonary embolism detection: prospective evaluation of dual-section helical CT versus selective pulmonary arteriography in 157 patients. Radiology 2000;217:447-55.
14 Perrier A, Howarth N, Didier D, et al. Performance of helical computed tomography in unselected outpatients with suspected pulmonary embolism. Ann Intern Med 2001;135:88-97.

15 Nilsson T, Soderberg $M$, Lundqvist $G$, et al. A comparison of spiral computed tomography and latex agglutination D-dimer assay in acute pulmonary embolism using pulmonary arteriography as gold standard. Scand Cardiovasc J 2002;36:373-7.

16 Hogg K, Dawson D, Tabor T, et al. Respiratory dead space measurement in the investigation of pulmonary embolism in outpatients with pleuritic chest pain. Chest 2005;128:2195-202.

17 Wells PS, Anderson DR, Rodger M, et al. Derivation of a simple clinical model to categorize patients probability of pulmonary embolism: increasing the models utility with the SimpliRED D-dimer. Thromb Haemost 2000;83:416-20

18 Newcombe RG. Two-sided confidence intervals for the single proportion: comparison of seven methods. Stat Med 1998;17:857-72.

19 Agresti A, Coull BA. Approximate is better than "exact" for interval estimation of binomial proportions. Am Stat 1998;52:119-26.

20 Stein PD. Clinical, laboratory, roentgenographic and electrocardiographic findings in patients with acute pulmonary embolism and no pre-existing cardiac or pulmonary disease. Chest 1991;100:598-603.

21 Hull RD, Raskob GE, Carter CJ, et al. Pulmonary embolism in outpatients with pleuritic chest pain. Arch Intern Med 1988;148:838-44. 